\title{
Effect of Storage Conditions and Packaging on Sensory Evaluation of Rice
}

\author{
D. Saida Naik $^{1^{*}}$ and M.B. Chetti ${ }^{2}$ \\ ${ }^{1}$ Department of Crop Physiology, College of Agriculture, Rajendranagar, \\ Hyderabad-500030, Telangana, India \\ ${ }^{2}$ Assistant Director General (HRD), Educational Division, ICAR, Pusa, \\ New Delhi-110012, India \\ *Corresponding author
}

A B S T R A C T

K e y w o r d s
$\begin{aligned} & \text { Sensory evaluation } \\ & \text { of cooked rice, } \\ & \text { Vacuum packaging } \\ & \text { and storage. }\end{aligned}$
Article Info
$\begin{aligned} & \text { Accepted: } \\ & \text { 10 September } 2017 \\ & \text { Available Online: } \\ & \text { 10 October } 2017\end{aligned}$

\section{Introduction}

Rice is primarily a high energy caloric food. The major part of rice consists of carbohydrate in the form of starch, which is about $72-75$ per cent of the total grain composition. The protein content of rice is around 7 per cent, which is mainly glutelin, also known as oryzenin. During storage a number of physicochemical and physiological changes occur, this is usually termed ageing. These changes which include pasting properties like colour, flavour and composition. As rice ages cooked rice texture becomes fluffier and harder. Optimum cooking time for milled rice was 4-6 minutes longer after 6 months of storage than it was at harvest. Sensory evaluation techniques have been used by several researchers to evaluate the effects of storage on end-use quality of rice.

Therefore, a scientific study on proper long term storage of grains to avoid rice quality deterioration is very much needed. Hence, the investigation on the physiological and biochemical changes during long term storage of rice grains under different packaging materials stored both under ambient as well as cold storage. 


\section{Materials and Methods}

A storage experiment was carried out for a period of 18 months at Department of Crop Physiology, University of Agricultural Sciences, Dharwad. Freshly harvested paddy seeds (BPT-5204) were dried under sun and milled stored under different storage conditions and containers. The temperature maintained in the cold storage was around (4 ${ }^{\circ} \mathrm{C} \pm 1{ }^{\circ} \mathrm{C}$ ) and relative humidity was 85 to 90 per cent. For ambient storage, bags were stored in the laboratory at room temperature $\left(25 \pm 2{ }^{\circ} \mathrm{C}\right)$.

Rice grains were packed in $100 \mathrm{~g}$ vacuum packed bags (The machine used for vacuum packaging of different grains was OLPACK $501 / \mathrm{V}$ manufactured by INTERPRISEBRUSSELS S.A., BRUXTAINER DIVISION, Belgium) and polythene bags while $5 \mathrm{~kg}$ rice was packed in cloth bags and gunny bags. After packaging of all the grains in different containers, $50 \%$ bags were stored properly in the iron racks without stacking so that all the bags were uniformly exposed to the particular treatment condition; while 50\% bags were stored under cold storage.

The treatment consisting of different containers viz., vacuum packed bags, polythene bags, cloth bags and gunny bags were replicated thrice in both cold and ambient storage conditions in completely randomised design with factorial concept.

\section{Sensory evaluation}

$50 \mathrm{~g}$ of rice samples were used for sensory evaluation in cooked form to a panel of semitrained judges who evaluated parameters appearance, color, flavour, texture, taste and overall acceptability of cooked rice. Following nine point hedonic scales was used to evaluate the samples (Amerine et al., 1965).

\section{Organoleptic score rating}

9 ---- Like extremely

8 ---- Like very much

7 ---- Like moderately

6 ---- Like slightly

5 ---- Neither like nor dislike

4 ---- Dislike slightly

3 ---- Dislike moderately

2 ---- Dislike very much

1 ---- Dislike extremely

Fisher's method of analysis of variance was applied for the analysis and interpretation of the experimental data as suggested by Panse and Sukhatme (1967) and level of significance used in ' $\mathrm{F}$ ' and ' $\mathrm{t}$ ' test was $P=$ 0.01 .

\section{Results and Discussion}

The data on appearance as influenced by storage containers, storage conditions and their interaction presented in Table 1. It was observed that score for appearance of cooked rice was decreased with increase in storage period among all the treatments from 2 months of storage and up to 18 months of storage. Among the containers, vacuum packaged bags $\left(\mathrm{C}_{1}\right)$ recorded higher score for appearance compared to all other treatments under both ambient storage $\left(\mathrm{S}_{1}\right)$ and cold storage $\left(\mathrm{S}_{2}\right)$. Lower score for appearance was observed in gunny bags $\left(\mathrm{C}_{4}\right)$ under both ambient storage $\left(S_{1}\right)$ and cold storage $\left(S_{2}\right)$ followed by cloth bags $\left(\mathrm{C}_{3}\right)$.

At $6^{\text {th }}$ months of storage, higher score for appearance was observed in vacuum packed bags stored under ambient condition $\left(\mathrm{C}_{1} \mathrm{~S}_{1}\right)$ (8.0) followed by vacuum packed bags stored under cold storage $\left(\mathrm{C}_{1} \mathrm{~S}_{2}\right)$ (7.8). The lower score for appearance was observed in gunny bags stored under ambient storage $\left(\mathrm{C}_{4} \mathrm{~S}_{1}\right)$ (7.2) followed by gunny bags stored under cold storage $\left(\mathrm{C}_{4} \mathrm{~S}_{2}\right)$ (6.6). A similar trend 
continued up to 18 months of storage. During the 18 months of storage, vacuum packaged bags $\left(\mathrm{C}_{1}\right)$ recorded significantly higher score, which was significantly higher compared to all other treatments under ambient storage $\left(\mathrm{S}_{1}\right)$ and cold storage $\left(\mathrm{S}_{2}\right)$. It was further noticed that, ambient storage $\left(\mathrm{S}_{1}\right)$ recorded higher score for appearance compared to cold storage $\left(\mathrm{S}_{2}\right)$ throughout the storage.

The observations on colour as influenced by different packaging and storage conditions differed between treatments (Table 2). The score for colour of cooked rice was decreased with advancement in storage period between the treatments and storage conditions and their interaction from 2 months of storage and up to 18 months of storage. Among containers, gunny bags $\left(\mathrm{C}_{4}\right)$ recorded lower score for colour compared over all other treatments under both ambient storage $\left(\mathrm{S}_{1}\right)$ and cold storage $\left(S_{2}\right)$. Significantly higher score for colour was observed in vacuum packaged bags $\left(\mathrm{C}_{1}\right)$ under both ambient storage $\left(\mathrm{S}_{1}\right)$ and cold storage $\left(\mathrm{S}_{2}\right)$ followed by polythene bags $\left(\mathrm{C}_{2}\right)$ at all the stages of storage.

At $6^{\text {th }}$ months of storage, higher score for colour was observed in vacuum packed bags stored under ambient condition $\left(\mathrm{C}_{1} \mathrm{~S}_{1}\right)(8.0)$ followed by vacuum packed bags stored under cold storage $\left(\mathrm{C}_{1} \mathrm{~S}_{2}\right)$ (7.6). The lower score for appearance was observed in gunny bags stored under ambient storage $\left(\mathrm{C}_{4} \mathrm{~S}_{1}\right)$ (7.2) followed by gunny bags stored under cold storage $\left(\mathrm{C}_{4} \mathrm{~S}_{2}\right)$ (6.7). A similar trend continued at $8,10,12,14,16$ and 18 months of storage. At 18 months of storage, vacuum packaged bags $\left(\mathrm{C}_{1}\right)$ recorded higher score for colour and lower score in gunny bags $\left(\mathrm{C}_{4}\right)$ compared to all other treatments under ambient storage $\left(S_{1}\right)$ and cold storage $\left(S_{2}\right)$. Among the storage conditions, ambient storage $\left(\mathrm{S}_{1}\right)$ showed higher score for colour compared to cold storage $\left(\mathrm{S}_{2}\right)$ among all the treatments.
The results on texture as influenced by storage containers, storage conditions and their interaction presented in Table 3 indicated differences between the storage containers and their interaction at all the stages of storage period. The decrease trend was observed with progress in storage period among all the treatments under both ambient storage $\left(\mathrm{S}_{1}\right)$ and cold storage $\left(\mathrm{S}_{2}\right)$. The higher score for texture of cooked rice was observed in vacuum packaged bags $\left(\mathrm{C}_{1}\right)$ under both ambient storage $\left(S_{1}\right)$ and cold storage $\left(S_{2}\right)$. Among the containers, Gunny bags $\left(\mathrm{C}_{4}\right)$ recorded lower score for texture compared to all other treatments under both ambient storage $\left(\mathrm{S}_{1}\right)$ and cold storage $\left(\mathrm{S}_{2}\right)$.

At 6 months of storage, the lower score for texture was observed in gunny bags stored under ambient storage $\left(\mathrm{C}_{4} \mathrm{~S}_{1}\right)$ (7.2) followed by gunny bags stored under cold storage $\left(\mathrm{C}_{4} \mathrm{~S}_{2}\right)$ (6.6). The higher score for texture was found in vacuum packed bags stored under ambient condition $\left(\mathrm{C}_{1} \mathrm{~S}_{1}\right)$ (7.8) followed by vacuum packed bags stored under cold storage $\left(\mathrm{C}_{1} \mathrm{~S}_{2}\right)$ (7.7), which was higher over all other treatments. A similar trend continued from 8 months and up to 18 months of storage. At 18 months of storage, vacuum packaged bags $\left(\mathrm{C}_{1}\right)$ recorded significantly higher score for colour and significantly lower score was observed in gunny bags $\left(\mathrm{C}_{4}\right)$ compared to other treatments under ambient storage $\left(S_{1}\right)$ and cold storage $\left(S_{2}\right)$. Among storage conditions, ambient storage $\left(\mathrm{S}_{1}\right)$ recorded higher score for colour compared to cold storage $\left(\mathrm{S}_{2}\right)$.

Effect of storage conditions and packaging and on flavor as shown in Table 4. The score for flavor of cooked rice decreased with an increase in storage period among all the treatments.

Among the storage containers, vacuum packaged bags $\left(\mathrm{C}_{1}\right)$ recorded higher score for 
flavour compared to all other treatments under both ambient storage $\left(\mathrm{S}_{1}\right)$ and cold storage $\left(S_{2}\right)$. Lower score was observed for flavour in gunny bags $\left(\mathrm{C}_{4}\right)$ under both ambient storage $\left(\mathrm{S}_{1}\right)$ and cold storage $\left(\mathrm{S}_{2}\right)$ followed by cloth bags $\left(\mathrm{C}_{3}\right)$. No much difference was observed between ambient storage $\left(\mathrm{S}_{1}\right)$ and cold storage $\left(\mathrm{S}_{2}\right)$ under vacuum packaged bags $\left(\mathrm{C}_{1}\right)$ followed by polythene bags $\left(\mathrm{C}_{2}\right)$.

Table.1 Effect of storage conditions and packaging on appearance at different periods of storage in rice grains

\begin{tabular}{|c|c|c|c|c|c|c|c|c|c|c|}
\hline \multirow{2}{*}{ Treatments } & \multicolumn{10}{|c|}{ Storage period (months) } \\
\hline & $\mathbf{0}$ & 2 & 4 & 6 & 8 & 10 & 12 & 14 & 16 & 18 \\
\hline \multicolumn{11}{|c|}{ Storage conditions mean (S) } \\
\hline$S_{1}$ & 8.0 & 7.8 & 7.7 & 7.6 & 7.4 & 7.3 & 6.9 & 6.8 & 6.6 & 6.2 \\
\hline $\mathbf{S}_{2}$ & 7.9 & 7.4 & 7.3 & 7.3 & 7.0 & 6.9 & 6.8 & 6.7 & 6.3 & 6.0 \\
\hline \multicolumn{11}{|c|}{ Storage containers mean $(\mathrm{C})$} \\
\hline $\mathrm{C}_{1}$ & 8.2 & 8.0 & 8.0 & 7.9 & 7.7 & 7.5 & 7.2 & 7.2 & 7.0 & 7.0 \\
\hline $\mathbf{C}_{2}$ & 8.1 & 7.8 & 7.8 & 7.8 & 7.5 & 7.4 & 7.1 & 6.9 & 6.7 & 6.4 \\
\hline $\mathbf{C}_{3}$ & 7.8 & 7.5 & 7.4 & 7.1 & 7.0 & 6.9 & 6.7 & 6.5 & 6.3 & 5.7 \\
\hline $\mathrm{C}_{4}$ & 7.7 & 7.1 & 6.9 & 6.9 & 6.6 & 6.6 & 6.3 & 6.3 & 5.9 & 5.3 \\
\hline \multicolumn{11}{|c|}{ Interaction mean $(\mathrm{S} \times \mathrm{C})$} \\
\hline$S_{1} \times C_{1}$ & 8.2 & 8.0 & 8.1 & 8.0 & 7.8 & 7.6 & 7.0 & 7.0 & 7.0 & 7.0 \\
\hline$S_{1} \times C_{2}$ & 8.1 & 8.0 & 8.0 & 7.8 & 7.4 & 7.2 & 7.0 & 7.0 & 6.6 & 6.0 \\
\hline $\mathrm{S}_{1} \times \mathrm{C}_{3}$ & 7.8 & 7.8 & 7.6 & 7.4 & 7.4 & 7.4 & 7.0 & 6.6 & 6.5 & 6.2 \\
\hline$S_{1} \times C_{4}$ & 7.8 & 7.4 & 7.2 & 7.2 & 6.8 & 6.8 & 6.4 & 6.4 & 6.2 & 5.5 \\
\hline$S_{2} \times C_{1}$ & 8.2 & 8.0 & 7.9 & 7.8 & 7.5 & 7.4 & 7.4 & 7.4 & 7.0 & 7.0 \\
\hline$S_{2} \times C_{2}$ & 8.0 & 7.6 & 7.6 & 7.8 & 7.6 & 7.5 & 7.2 & 6.8 & 6.8 & 6.8 \\
\hline$S_{2} \times C_{3}$ & 7.7 & 7.2 & 7.2 & 6.8 & 6.6 & 6.4 & 6.4 & 6.4 & 6.0 & 5.2 \\
\hline$S_{2} \times C_{4}$ & 7.6 & 6.8 & 6.6 & 6.6 & 6.4 & 6.4 & 6.2 & 6.2 & 5.5 & 5.0 \\
\hline $\begin{array}{l}\text { Grand } \\
\text { Mean }\end{array}$ & 7.9 & 7.6 & 7.5 & 7.4 & 7.2 & 7.1 & 6.8 & 6.7 & 6.5 & 6.1 \\
\hline \multicolumn{11}{|l|}{ S.Em \pm} \\
\hline$\overline{\mathbf{S}}$ & 0.21 & 0.31 & 0.26 & 0.30 & 0.25 & 0.25 & 0.26 & 0.26 & 0.27 & 0.32 \\
\hline $\mathrm{C}$ & 0.30 & 0.22 & 0.18 & 0.21 & 0.35 & 0.35 & 0.37 & 0.36 & 0.39 & 0.45 \\
\hline $\mathbf{S} \times \mathbf{C}$ & 0.43 & 0.44 & 0.37 & 0.42 & 0.36 & 0.49 & 0.53 & 0.52 & 0.55 & 1.30 \\
\hline \multicolumn{11}{|l|}{ C.D. (1\%) } \\
\hline $\mathbf{S}$ & NS & NS & NS & NS & NS & NS & NS & NS & NS & $\mathrm{NS}$ \\
\hline C & NS & NS & NS & NS & NS & NS & NS & NS & 1.12 & 1.29 \\
\hline $\mathbf{S} \times \mathbf{C}$ & $\mathrm{NS}$ & NS & NS & NS & NS & NS & NS & NS & NS & $\mathrm{NS}$ \\
\hline & & & & & & & & & & \\
\hline $\begin{array}{l}1=\text { Ambient stora } \\
2=\text { Cold storage }\end{array}$ & & & $\begin{array}{l}=\text { Vacu } \\
=\text { Polyt }\end{array}$ & $\begin{array}{l}m \text { packe } \\
\text { ene bag }\end{array}$ & bags & & $\begin{array}{l}=\text { Clotl } \\
=\text { Gun! }\end{array}$ & $\begin{array}{l}\operatorname{ggs} \\
\mathrm{bags}\end{array}$ & & \\
\hline
\end{tabular}


Table.2 Effect of storage conditions and packaging on colour at different periods of storage in rice grains

\begin{tabular}{|c|c|c|c|c|c|c|c|c|c|c|}
\hline \multirow{2}{*}{ Treatments } & \multicolumn{10}{|c|}{ Storage period (months) } \\
\hline & $\mathbf{0}$ & 2 & 4 & 6 & 8 & 10 & 12 & 14 & 16 & 18 \\
\hline \multicolumn{11}{|c|}{ Storage conditions mean $(\mathrm{S})$} \\
\hline$S_{1}$ & 8.0 & 7.8 & 7.7 & 7.6 & 7.4 & 7.3 & 7.1 & 6.9 & 6.4 & 6.2 \\
\hline $\mathbf{S}_{\mathbf{2}}$ & 7.9 & 7.6 & 7.5 & 7.4 & 7.2 & 7.1 & 6.9 & 6.8 & 6.4 & 6.1 \\
\hline \multicolumn{11}{|c|}{ Storage containers mean $(\mathrm{C})$} \\
\hline $\mathbf{C}_{1}$ & 8.1 & 8.1 & 7.9 & 7.8 & 7.8 & 7.7 & 7.5 & 7.4 & 7.2 & 7.0 \\
\hline $\mathbf{C}_{2}$ & 8.0 & 7.9 & 7.7 & 7.6 & 7.5 & 7.5 & 7.4 & 7.2 & 6.9 & 6.6 \\
\hline $\mathbf{C}_{3}$ & 7.9 & 7.7 & 7.6 & 7.3 & 7.2 & 7.0 & 6.8 & 7.0 & 6.0 & 5.8 \\
\hline $\mathrm{C}_{4}$ & 7.9 & 7.1 & 7.0 & 7.0 & 6.8 & 6.6 & 6.6 & 6.2 & 5.8 & 5.4 \\
\hline \multicolumn{11}{|c|}{ Interaction mean $(\mathrm{S} \times \mathrm{C})$} \\
\hline$S_{1} \times C_{1}$ & 8.1 & 8.2 & 8.0 & 8.0 & 7.9 & 7.8 & 7.6 & 7.5 & 7.2 & 7.0 \\
\hline$S_{1} \times C_{2}$ & 8.0 & 8.0 & 7.8 & 7.8 & 7.6 & 7.6 & 7.5 & 7.4 & 7.1 & 6.9 \\
\hline $\mathbf{S}_{1} \times \mathbf{C}_{3}$ & 7.9 & 7.8 & 7.8 & 7.8 & 7.6 & 7.6 & 7.3 & 7.0 & 6.2 & 6.3 \\
\hline $\mathrm{S}_{1} \times \mathrm{C}_{4}$ & 8.0 & 7.4 & 7.2 & 7.2 & 7.0 & 6.8 & 6.6 & 6.2 & 6.1 & 5.5 \\
\hline $\mathbf{S}_{2} \times \mathbf{C}_{1}$ & 8.0 & 8.0 & 7.8 & 7.6 & 7.6 & 7.5 & 7.3 & 7.2 & 7.1 & 7.0 \\
\hline $\mathbf{S}_{2} \times C_{2}$ & 8.0 & 7.8 & 7.6 & 7.4 & 7.4 & 7.4 & 7.2 & 7.0 & 6.6 & 6.2 \\
\hline$S_{2} \times C_{3}$ & 7.8 & 7.5 & 7.3 & 6.8 & 6.8 & 6.4 & 6.2 & 7.0 & 5.8 & 5.2 \\
\hline$S_{2} \times C_{4}$ & 7.8 & 6.8 & 6.8 & 6.7 & 6.6 & 6.4 & 6.5 & 6.2 & 5.5 & 5.2 \\
\hline $\begin{array}{l}\text { Grand } \\
\text { Mean }\end{array}$ & 8.0 & 7.7 & 7.5 & 7.4 & 7.3 & 7.2 & 7.0 & 6.9 & 6.5 & 6.2 \\
\hline \multicolumn{11}{|l|}{$\mathbf{S . E m} \pm$} \\
\hline $\mathbf{S}$ & 0.22 & 0.23 & 0.18 & 0.20 & 0.21 & 0.18 & 0.19 & 0.21 & 0.21 & 0.21 \\
\hline C & 0.31 & 0.33 & 0.26 & 0.29 & 0.30 & 0.25 & 0.27 & 0.29 & 0.30 & 0.29 \\
\hline $\mathbf{S} \times \mathbf{C}$ & 0.44 & 0.47 & 0.37 & 0.41 & 0.43 & 0.36 & 0.39 & 0.42 & 0.43 & 0.42 \\
\hline \multicolumn{11}{|l|}{ C.D. (1\%) } \\
\hline $\mathbf{S}$ & NS & NS & NS & NS & NS & NS & NS & NS & NS & NS \\
\hline C & NS & NS & NS & NS & NS & NS & NS & 0.86 & 0.88 & 0.86 \\
\hline $\mathbf{S} \times \mathbf{C}$ & NS & NS & NS & NS & NS & NS & NS & NS & NS & NS \\
\hline
\end{tabular}


Table.3 Effect of storage conditions and packaging on texture at different periods of storage in rice grains

\begin{tabular}{|c|c|c|c|c|c|c|c|c|c|c|}
\hline \multirow{2}{*}{ Treatments } & \multicolumn{10}{|c|}{ Storage period (months) } \\
\hline & $\mathbf{0}$ & 2 & 4 & 6 & 8 & 10 & 12 & 14 & 16 & 18 \\
\hline \multicolumn{11}{|c|}{ Storage conditions mean $(\mathrm{S})$} \\
\hline $\mathbf{S}_{\mathbf{1}}$ & 8.0 & 7.6 & 7.4 & 7.4 & 7.3 & 7.6 & 7.5 & 7.5 & 7.4 & 7.4 \\
\hline $\mathbf{S}_{2}$ & 8.0 & 7.6 & 7.3 & 7.2 & 7.1 & 7.6 & 7.5 & 7.5 & 7.2 & 6.5 \\
\hline \multicolumn{11}{|c|}{ Storage containers mean $(C)$} \\
\hline $\mathbf{C}_{1}$ & 8.1 & 8.0 & 7.8 & 7.8 & 7.7 & 7.6 & 7.5 & 7.5 & 7.3 & 7.0 \\
\hline $\mathbf{C}_{2}$ & 8.0 & 7.9 & 7.6 & 7.5 & 7.5 & 7.2 & 7.0 & 7.0 & 6.5 & 6.3 \\
\hline $\mathrm{C}_{3}$ & 7.9 & 7.4 & 7.1 & 7.0 & 6.8 & 6.7 & 6.4 & 6.1 & 5.8 & 5.6 \\
\hline $\mathbf{C}_{4}$ & 8.0 & 7.2 & 7.0 & 6.9 & 6.9 & 6.7 & 6.2 & 5.9 & 5.7 & 5.4 \\
\hline \multicolumn{11}{|c|}{ Interaction mean $(\mathrm{S} \times \mathrm{C})$} \\
\hline$S_{1} \times C_{1}$ & 8.1 & 8.0 & 7.8 & 7.8 & 7.7 & 7.6 & 7.5 & 7.5 & 7.4 & 7.4 \\
\hline$S_{1} \times C_{2}$ & 8.0 & 8.0 & 7.6 & 7.5 & 7.5 & 7.2 & 7.1 & 7.0 & 6.8 & 6.5 \\
\hline$S_{1} \times C_{3}$ & 7.9 & 7.6 & 7.2 & 7.1 & 7.0 & 7.0 & 6.7 & 6.6 & 6.1 & 6.0 \\
\hline$S_{1} \times C_{4}$ & 8.0 & 7.3 & 7.2 & 7.2 & 7.1 & 7.0 & 6.5 & 6.2 & 6.1 & 5.6 \\
\hline$S_{2} \times C_{1}$ & 8.0 & 8.0 & 7.8 & 7.7 & 7.6 & 7.6 & 7.5 & 7.5 & 7.2 & 6.5 \\
\hline$S_{2} \times C_{2}$ & 8.0 & 7.8 & 7.5 & 7.4 & 7.4 & 7.2 & 6.8 & 7.0 & 6.2 & 6.0 \\
\hline$S_{2} \times C_{3}$ & 7.9 & 7.2 & 6.9 & 6.8 & 6.6 & 6.3 & 6.1 & 5.6 & 5.5 & 5.2 \\
\hline$S_{2} \times C_{4}$ & 7.9 & 7.0 & 6.8 & 6.6 & 6.6 & 6.4 & 5.8 & 5.5 & 5.2 & 5.2 \\
\hline $\begin{array}{c}\text { Grand } \\
\text { Mean }\end{array}$ & 8.0 & 7.6 & 7.4 & 7.3 & 7.2 & 7.0 & 6.8 & 6.6 & 6.3 & 6.1 \\
\hline \multicolumn{11}{|l|}{ S.Em \pm} \\
\hline $\mathbf{S}$ & 0.22 & 0.19 & 0.19 & 0.23 & 0.23 & 0.20 & 0.19 & 0.22 & 0.21 & 0.22 \\
\hline C & 0.32 & 0.27 & 0.27 & 0.32 & 0.32 & 0.28 & 0.28 & 0.32 & 0.30 & 0.32 \\
\hline $\mathbf{S} \times \mathbf{C}$ & 0.45 & 0.38 & 0.37 & 0.45 & 0.45 & 0.40 & 0.39 & 0.45 & 0.42 & 0.45 \\
\hline \multicolumn{11}{|l|}{ C.D. (1\%) } \\
\hline $\mathbf{S}$ & NS & NS & NS & NS & NS & NS & NS & NS & NS & NS \\
\hline C & NS & NS & NS & NS & NS & NS & NS & 0.92 & 0.85 & 0.92 \\
\hline $\mathrm{S} \times \mathrm{C}$ & NS & NS & NS & NS & NS & NS & NS & NS & NS & NS \\
\hline
\end{tabular}


Table.4 Effect of storage conditions and packaging on flavor at different periods of storage in rice grains

\begin{tabular}{|c|c|c|c|c|c|c|c|c|c|c|}
\hline \multirow{2}{*}{ Treatments } & \multicolumn{10}{|c|}{ Storage period (months) } \\
\hline & $\mathbf{0}$ & 2 & 4 & 6 & 8 & 10 & 12 & 14 & 16 & 18 \\
\hline \multicolumn{11}{|c|}{ Storage conditions mean $(\mathbf{S})$} \\
\hline $\mathbf{S}_{1}$ & 8.0 & 7.8 & 7.5 & 7.3 & 7.4 & 7.0 & 6.8 & 6.6 & 6.5 & 6.4 \\
\hline $\mathbf{S}_{\mathbf{2}}$ & 7.9 & 7.4 & 7.3 & 7.1 & 7.1 & 6.8 & 6.7 & 6.4 & 6.0 & 5.7 \\
\hline \multicolumn{11}{|c|}{ Storage containers mean $(\mathrm{C})$} \\
\hline $\mathbf{C}_{1}$ & 8.1 & 8.2 & 7.9 & 7.8 & 7.8 & 7.7 & 7.5 & 7.5 & 7.3 & 7.2 \\
\hline $\mathbf{C}_{2}$ & 8 & 7.9 & 7.7 & 7.3 & 7.3 & 6.8 & 6.7 & 6.4 & 6.0 & 5.8 \\
\hline $\mathrm{C}_{3}$ & 7.9 & 7.4 & 7.2 & 7.1 & 7.1 & 6.6 & 6.5 & 6.1 & 5.8 & 5.7 \\
\hline $\mathbf{C}_{4}$ & 7.9 & 7.2 & 6.8 & 6.7 & 6.7 & 6.6 & 6.4 & 6.2 & 5.9 & 5.6 \\
\hline \multicolumn{11}{|c|}{ Interaction mean $(\mathrm{S} \times \mathrm{C})$} \\
\hline$S_{1} \times C_{1}$ & 8.1 & 8.2 & 8 & 7.8 & 7.8 & 7.6 & 7.6 & 7.5 & 7.4 & 7.3 \\
\hline$S_{1} \times C_{2}$ & 8.0 & 8.0 & 7.7 & 7.4 & 7.4 & 7.0 & 6.8 & 6.4 & 6.4 & 6.2 \\
\hline$S_{1} \times C_{3}$ & 8.0 & 7.5 & 7.4 & 7.3 & 7.3 & 6.6 & 6.5 & 6.2 & 6.2 & 6.2 \\
\hline $\mathrm{S}_{1} \times \mathrm{C}_{4}$ & 7.9 & 7.2 & 7.0 & 6.9 & 6.9 & 6.8 & 6.4 & 6.3 & 6.0 & 6.0 \\
\hline$S_{2} \times C_{1}$ & 8.1 & 8.0 & 7.8 & 7.7 & 7.7 & 7.7 & 7.4 & 7.4 & 7.2 & 7.0 \\
\hline$S_{2} \times C_{2}$ & 7.9 & 7.8 & 7.6 & 7.2 & 7.2 & 6.6 & 6.5 & 6.3 & 5.6 & 5.4 \\
\hline$S_{2} \times C_{3}$ & 7.8 & 7.2 & 7.0 & 6.9 & 6.9 & 6.5 & 6.4 & 6.0 & 5.4 & 5.2 \\
\hline $\mathrm{S}_{2} \times \mathrm{C}_{4}$ & 7.9 & 7.0 & 6.6 & 6.5 & 6.5 & 6.4 & 6.4 & 6.0 & 5.8 & 5.2 \\
\hline $\begin{array}{c}\text { Grand } \\
\text { Mean }\end{array}$ & 8.0 & 7.6 & 7.4 & 7.2 & 7.2 & 6.9 & 6.8 & 6.5 & 6.3 & 6.1 \\
\hline \multicolumn{11}{|l|}{ S.Em \pm} \\
\hline $\mathbf{S}$ & 0.22 & 0.29 & 0.28 & 0.28 & 0.27 & 0.27 & 0.25 & 0.25 & 0.26 & 0.26 \\
\hline C & 0.32 & 0.43 & 0.42 & 0.41 & 0.40 & 0.40 & 0.39 & 0.40 & 0.42 & 0.42 \\
\hline $\mathrm{S} \times \mathrm{C}$ & 0.45 & 0.58 & 0.57 & 0.57 & 0.55 & 0.55 & 0.53 & 0.55 & 0.58 & 0.58 \\
\hline \multicolumn{11}{|l|}{ C.D. (1\%) } \\
\hline $\mathbf{S}$ & NS & NS & NS & NS & NS & NS & NS & NS & NS & NS \\
\hline $\mathbf{C}$ & NS & NS & NS & NS & NS & NS & NS & 1.16 & 1.21 & 1.30 \\
\hline $\mathrm{S} \times \mathrm{C}$ & NS & NS & NS & NS & NS & NS & NS & NS & NS & NS \\
\hline
\end{tabular}


Table.5 Effect of storage conditions and packaging on taste at different periods of storage in rice grains

\begin{tabular}{|c|c|c|c|c|c|c|c|c|c|c|}
\hline \multirow{2}{*}{ Treatments } & \multicolumn{10}{|c|}{ Storage period (months) } \\
\hline & $\mathbf{0}$ & 2 & 4 & 6 & 8 & 10 & 12 & 14 & 16 & 18 \\
\hline \multicolumn{11}{|c|}{ Storage conditions mean (S) } \\
\hline $\mathbf{S}_{1}$ & 6.7 & 6.5 & 6.7 & 6.7 & 6.9 & 7.0 & 7.1 & 7.2 & 7.2 & 7.2 \\
\hline $\mathbf{S}_{\mathbf{2}}$ & 6.5 & 6.5 & 6.5 & 6.6 & 6.7 & 6.7 & 7.0 & 7.1 & 7.0 & 7.1 \\
\hline \multicolumn{11}{|c|}{ Storage containers mean $(C)$} \\
\hline $\mathbf{C}_{1}$ & 6.7 & 6.7 & 6.8 & 6.8 & 6.9 & 7.3 & 7.4 & 7.4 & 7.5 & 7.6 \\
\hline $\mathbf{C}_{2}$ & 6.6 & 6.5 & 6.5 & 6.7 & 6.8 & 7.0 & 7.1 & 7.2 & 7.3 & 7.4 \\
\hline $\mathrm{C}_{3}$ & 6.5 & 6.5 & 6.7 & 6.7 & 6.8 & 6.6 & 6.9 & 7.0 & 7.1 & 7.0 \\
\hline $\mathbf{C}_{4}$ & 6.7 & 6.5 & 6.5 & 6.6 & 6.6 & 6.6 & 6.8 & 6.9 & 6.7 & 6.6 \\
\hline \multicolumn{11}{|c|}{ Interaction mean $(S \times C)$} \\
\hline$S_{1} \times C_{1}$ & 6.8 & 6.7 & 6.8 & 6.9 & 7.0 & 7.4 & 7.4 & 7.5 & 7.6 & 7.7 \\
\hline$S_{1} \times C_{2}$ & 6.7 & 6.5 & 6.6 & 6.7 & 6.9 & 7.2 & 7.1 & 7.2 & 7.3 & 7.4 \\
\hline$S_{1} \times C_{3}$ & 6.5 & 6.5 & 6.7 & 6.7 & 6.8 & 6.7 & 7.0 & 7.1 & 7.2 & 7.0 \\
\hline$S_{1} \times C_{4}$ & 6.7 & 6.4 & 6.5 & 6.6 & 6.7 & 6.8 & 6.8 & 7.0 & 6.8 & 6.6 \\
\hline$S_{2} \times C_{1}$ & 6.6 & 6.6 & 6.7 & 6.7 & 6.8 & 7.2 & 7.3 & 7.3 & 7.3 & 7.4 \\
\hline$S_{2} \times C_{2}$ & 6.5 & 6.4 & 6.4 & 6.6 & 6.7 & 6.8 & 7.0 & 7.1 & 7.2 & 7.3 \\
\hline$S_{2} \times C_{3}$ & 6.4 & 6.5 & 6.6 & 6.6 & 6.7 & 6.5 & 6.8 & 6.9 & 7.0 & 7.0 \\
\hline$S_{2} \times C_{4}$ & 6.6 & 6.5 & 6.4 & 6.5 & 6.5 & 6.4 & 6.7 & 6.8 & 6.6 & 6.5 \\
\hline $\begin{array}{l}\text { Grand } \\
\text { Mean }\end{array}$ & 6.6 & 6.5 & 6.6 & 6.7 & 6.8 & 6.9 & 7.0 & 7.1 & 7.1 & 7.1 \\
\hline \multicolumn{11}{|l|}{ S.Em \pm} \\
\hline $\mathbf{S}$ & 0.22 & 0.19 & 0.19 & 0.23 & 0.23 & 0.20 & 0.19 & 0.22 & 0.21 & 0.22 \\
\hline C & 0.32 & 0.27 & 0.27 & 0.32 & 0.32 & 0.28 & 0.28 & 0.32 & 0.30 & 0.32 \\
\hline $\mathrm{S} \times \mathrm{C}$ & 0.45 & 0.38 & 0.37 & 0.45 & 0.45 & 0.40 & 0.39 & 0.45 & 0.42 & 0.45 \\
\hline \multicolumn{11}{|l|}{ C.D. (1\%) } \\
\hline $\mathbf{S}$ & NS & NS & NS & NS & NS & NS & NS & NS & NS & NS \\
\hline C & NS & NS & NS & NS & NS & NS & NS & NS & 0.85 & 0.92 \\
\hline $\mathrm{S} \times \mathrm{C}$ & NS & NS & NS & NS & NS & NS & NS & NS & NS & NS \\
\hline
\end{tabular}


Table.6 Effect of storage conditions and packaging on overall acceptability at different periods of storage in rice grains

\begin{tabular}{|c|c|c|c|c|c|c|c|c|c|c|}
\hline \multirow{2}{*}{ Treatments } & \multicolumn{10}{|c|}{ Storage period (months) } \\
\hline & $\mathbf{0}$ & 2 & 4 & 6 & 8 & 10 & 12 & 14 & 16 & 18 \\
\hline \multicolumn{11}{|c|}{ Storage conditions mean (S) } \\
\hline $\mathbf{S}_{1}$ & 7.8 & 7.8 & 7.6 & 7.4 & 7.4 & 7.2 & 7.0 & 6.9 & 6.6 & 6.2 \\
\hline $\mathbf{S}_{2}$ & 7.7 & 7.5 & 7.3 & 7.2 & 6.9 & 6.8 & 6.7 & 6.4 & 6.2 & 5.8 \\
\hline \multicolumn{11}{|c|}{ Storage containers mean $(\mathrm{C})$} \\
\hline $\mathbf{C}_{1}$ & 8.0 & 8.0 & 7.8 & 7.7 & 7.5 & 7.4 & 7.3 & 7.2 & 7.0 & 6.9 \\
\hline $\mathbf{C}_{2}$ & 7.8 & 7.8 & 7.7 & 7.5 & 7.3 & 7.2 & 7.0 & 7.0 & 6.6 & 6.2 \\
\hline $\mathbf{C}_{3}$ & 7.7 & 7.5 & 7.2 & 7.0 & 6.9 & 6.8 & 6.6 & 6.3 & 6.1 & 5.8 \\
\hline $\mathrm{C}_{4}$ & 7.7 & 7.4 & 7.0 & 7.0 & 6.8 & 6.7 & 6.5 & 6.2 & 5.8 & 5.1 \\
\hline \multicolumn{11}{|c|}{ Interaction mean (S x C) } \\
\hline$S_{1} \times C_{1}$ & 8.0 & 8.0 & 7.8 & 7.6 & 7.6 & 7.5 & 7.4 & 7.2 & 7.0 & 7.0 \\
\hline $\mathrm{S}_{1} \times \mathrm{C}_{2}$ & 7.9 & 8.0 & 7.8 & 7.5 & 7.4 & 7.2 & 7.0 & 7.0 & 6.8 & 6.4 \\
\hline $\mathrm{S}_{1} \times \mathrm{C}_{3}$ & 7.8 & 7.8 & 7.4 & 7.2 & 7.2 & 7.1 & 6.8 & 6.8 & 6.6 & 6.2 \\
\hline $\mathrm{S}_{1} \times \mathrm{C}_{4}$ & 7.6 & 7.4 & 7.2 & 7.2 & 7.2 & 7.0 & 6.8 & 6.6 & 6.2 & 5.6 \\
\hline $\mathbf{S}_{\mathbf{2}} \times \mathbf{C}_{\mathbf{1}}$ & 7.9 & 8.0 & 7.8 & 7.7 & 7.4 & 7.3 & 7.2 & 7.1 & 7.0 & 6.8 \\
\hline $\mathbf{S}_{2} \times \mathbf{C}_{2}$ & 7.7 & 7.6 & 7.6 & 7.4 & 7.2 & 7.2 & 7.0 & 7.0 & 6.4 & 6.0 \\
\hline $\mathbf{S}_{\mathbf{2}} \times \mathbf{C}_{\mathbf{3}}$ & 7.6 & 7.2 & 7.0 & 6.8 & 6.6 & 6.4 & 6.4 & 5.8 & 5.6 & 5.4 \\
\hline $\mathrm{S}_{2} \times \mathrm{C}_{4}$ & 7.7 & 7.3 & 6.8 & 6.8 & 6.4 & 6.4 & 6.2 & 5.8 & 5.4 & 4.5 \\
\hline Grand Mean & 7.8 & 7.7 & 7.4 & 7.3 & 7.1 & 7.0 & 6.9 & 6.7 & 6.4 & 6.0 \\
\hline \multicolumn{11}{|l|}{ S.Em \pm} \\
\hline $\mathbf{S}$ & 0.17 & 0.19 & 0.22 & 0.21 & 0.21 & 0.20 & 0.21 & 0.23 & 0.25 & 0.26 \\
\hline $\mathbf{C}$ & 0.25 & 0.27 & 0.32 & 0.30 & 0.31 & 0.28 & 0.32 & 0.34 & 0.36 & 0.38 \\
\hline $\mathbf{S} \times \mathbf{C}$ & 0.35 & 0.38 & 0.45 & 0.44 & 0.44 & 0.40 & 0.43 & 0.46 & 0.49 & 0.50 \\
\hline \multicolumn{11}{|l|}{ C.D. $(1 \%)$} \\
\hline S & NS & NS & NS & NS & NS & NS & NS & NS & NS & NS \\
\hline $\mathbf{C}$ & NS & NS & NS & NS & NS & NS & NS & 0.94 & 1.13 & 1.17 \\
\hline $\mathbf{S} \times \mathbf{C}$ & NS & NS & NS & NS & NS & NS & NS & NS & NS & NS \\
\hline
\end{tabular}


At $8^{\text {th }}$ months of storage, higher score for flavor was observed in vacuum packed bags stored under ambient condition $\left(\mathrm{C}_{1} \mathrm{~S}_{1}\right)$ (7.8) followed by vacuum packed bags stored under cold storage $\left(\mathrm{C}_{1} \mathrm{~S}_{2}\right)(7.7)$, which was negligible score among the treatments. The lower score for flavor was observed in gunny bags stored under ambient storage $\left(\mathrm{C}_{4} \mathrm{~S}_{1}\right)$ (6.9) followed by gunny bags stored under cold storage $\left(\mathrm{C}_{4} \mathrm{~S}_{2}\right)(6.5)$, which was lower compared to all other treatments. A similar trend continued at 10,12, 14, 16 and 18 months of storage. At 18 months of storage, vacuum packaged bags $\left(\mathrm{C}_{1}\right)$ recorded significantly higher score for flavor, which was significantly higher over all other treatments under ambient storage $\left(\mathrm{S}_{1}\right)$ and cold storage $\left(\mathrm{S}_{2}\right)$. It was further noticed that, ambient storage $\left(\mathrm{S}_{1}\right)$ exhibited higher score for flavor compared to cold storage $\left(\mathrm{S}_{2}\right)$ among the treatments throughout the storage period.

The data on taste as influenced by storage containers, storage conditions presented in Table 5 indicated differences between treatments up to 18 months of storage. Among the containers, vacuum packaged bags $\left(\mathrm{C}_{1}\right)$ showed higher score for taste over all other treatments under both ambient storage $\left(S_{1}\right)$ and cold storage $\left(S_{2}\right)$. Lower score was observed for taste in gunny bags $\left(\mathrm{C}_{4}\right)$ followed by cloth bags $\left(\mathrm{C}_{3}\right)$. The score for taste of cooked rice increased with an advancement in storage period between the treatments under both ambient storage $\left(S_{1}\right)$ and cold storage $\left(\mathrm{S}_{2}\right)$ was observed.

At 8 months of storage, higher score for taste was observed in vacuum packed bags stored under ambient condition $\left(\mathrm{C}_{1} \mathrm{~S}_{1}\right)(6.9)$ followed by vacuum packed bags stored under cold storage $\left(\mathrm{C}_{1} \mathrm{~S}_{2}\right)$ (6.7) and lower score for taste was observed in gunny bags stored under ambient storage $\left(\mathrm{C}_{4} \mathrm{~S}_{1}\right)$ (6.4) followed by gunny bags stored under cold storage $\left(\mathrm{C}_{4} \mathrm{~S}_{2}\right)$
(6.0). A similar trend continued up to 18 months of storage. At end of the 18 months of storage, vacuum packaged bags $\left(\mathrm{C}_{1}\right)$ recorded significantly higher score (7.6) for taste, which was significantly superior over all other treatments under ambient storage $\left(\mathrm{S}_{1}\right)$ and cold storage $\left(\mathrm{S}_{2}\right)$. Lower score was observed in gunny bags stored under cold storage $\left(\mathrm{C}_{4} \mathrm{~S}_{2}\right)$ (6.5) followed by cloth bags stored under ambient storage $\left(\mathrm{C}_{4} \mathrm{~S}_{1}\right)$.

The observations on overall acceptability as influenced by different packaging and storage conditions measured up to 18 months as presented in Table 6 revealed differences among all the treatments, storage conditions and their interaction. Among the treatments, higher score for overall acceptability was found in vacuum packaged bags $\left(\mathrm{C}_{1}\right)$ followed polythene bags $\left(\mathrm{C}_{2}\right)$ under both ambient storage $\left(S_{1}\right)$ and cold storage $\left(S_{2}\right)$ which was almost negligible. The treatment gunny bags $\left(\mathrm{C}_{4}\right)$ recorded very low score for overall acceptability compared to all other treatments.

At 8 months of storage, score for overall acceptability was higher in vacuum packed bags stored under ambient condition $\left(\mathrm{C}_{1} \mathrm{~S}_{1}\right)$ (7.6) followed by vacuum packed bags stored under cold storage $\left(\mathrm{C}_{1} \mathrm{~S}_{2}\right)$ (7.4), which was higher over all other treatments. The lower score for overall acceptability was observed in gunny bags stored under ambient storage $\left(\mathrm{C}_{4} \mathrm{~S}_{1}\right)$ (7.2) followed by gunny bags stored under cold storage $\left(\mathrm{C}_{4} \mathrm{~S}_{2}\right)$ (6.4). At 18 months of storage, significantly higher score for overall acceptability was observed in vacuum packaged bags $\left(\mathrm{C}_{1}\right)$ (6.9), which was significantly higher compared to all other treatments. Significantly lower score for overall acceptability was seen in gunny bags $\left(\mathrm{C}_{4}\right)$ (5.1), which were significantly lower over all other treatments under ambient storage $\left(\mathrm{S}_{1}\right)$ and cold storage $\left(\mathrm{S}_{2}\right)$. Among storage conditions, ambient storage $\left(\mathrm{S}_{1}\right)$ 
recorded higher score for overall acceptability compared to cold storage $\left(\mathrm{S}_{2}\right)$ throughout the storage period.

Physical and chemical changes occur during storage. The eating and cooking properties are affected by the starch, protein and protein interaction, only structural changes occur rather than the change in the starch and protein interactions in rice grains. These structural changes affect the flavor, texture, taste and colour. Short term as well as long term storage has effect on rice pasting as well as cooking and eating characteristics, but the long term storage has the significant effect (Perdon et al., 1997).

Sensory evaluation of rice grains revealed that the as the storage period increased score for appearance, texture, colour and overall acceptability decreased, except the taste. Among the containers, vacuum packed bags recorded higher score for appearance, texture, colour, taste and overall acceptability compared to polythene bags, while gunny bags followed by cloth bags recorded lower score. This may be due to moisture pervious nature of gunny bags. The degradation was also related to the moisture content of the samples during storage. Samples with higher levels of moisture content degrade more rapidly than the lower levels of moisture content due to oxidation (Chiu et al., 2003).

When storage period increased, it affected the texture which may be due to physiochemical changes of rice during storage. Vacuum packaging reduced oxygen level in the packs compared to gunny bags and the anaerobic environment thus created prevents the growth of spoilage microorganism's especially aerobic ones which are responsible for off odor, slime and texture changes. Similar observations were reported by Nunez et al., 1986), Indhudhara Swamy et al., (1978) and Chrastil et al., (1992). The score for taste was decreased with an increased storage period. Vacuum packed seeds recorded higher score for taste compared to polythene bags, while lower score for gunny bags and cloth bags. Enhanced storage period increases the taste which is more acceptable than fresh rice (Meuellenet et al., 1999). In the present investigation aroma, flavour and colour for cooked rice decreased with advancement in storage period. The scores for aroma, flavour and colour were higher in vacuum packed bags, while lower in gunny bags followed by cloth bags. Similar observations were made by Sidik (2000).

Sensory evaluation of rice grains revealed that appearance, texture, colour and overall acceptability values decreased with an increase in storage period, except the taste. Among the containers, vacuum packed bags recorded higher score for appearance, texture, colour, taste and overall acceptability compared to polythene bags; while gunny bags recorded lower score followed by cloth bags.

\section{References}

Amerine, M. A., Pangborn, R. M. and Roessler, E. B., 1965. Principles of Sensory Evaluation of Food, Academic Press, London.

Chiu, K. Y., Chem, C. L. and Sung, J. M., 2003. Partial vacuum storage improves the longevity of primed Sh-2 sweet corn seeds. Sci Hort., 98 (2) : 99-111.

Chrastil, J., 1992. Correlations between the physico chemical and functional properties of rice. J. Agric. Food Chem., 40: 1683-1686.

Indudhara Swamy, Y. M., Sowbhagya, C. M. and Bhattacharya, K. R., 1978. Changes in the physicochemical properties of rice with ageing. J. Sci. Food Agric., 29: 627-639.

Meuellenet, J. F. C., Marks, B. P., Griffin, K. 
and Daniels, M. J., 1999. Effects of rough rice drying and storage conditions on sensory profiles of cooked rice. Cereal Chem., 76: 483-486.

Nunez, M. P., Gaya, M., Madena, M., Rodriguezmarian, M. A. and GarciaAcer, C., 1986. Changes in microbiological, chemical, rheological and sensory characteristics during ripening of vacuum packaged Manchego cheese. J. Food Sci., 21 (2) : 115-123.
Panse, V. G., and Sukhatme, P. V., 1967. Statistical Methods for Agricultural Workers, Indian Council of Agricultural Research, New Delhi, pp. 167-174

Perdon, A. A., Marks, B. P., Siebenmorgen, T. J. and Reid, N. B., 1997. Effects of rough rice storage conditions on the amylograph and cooking properties of medium-grain rice. Bengal. J. Cereal Chem., 74 : 864-867.

Sidik, S., 2000. The quality changes of rice stored under vacuum conditions. $J$. Agric. Technol., 1(3): 55-63.

\section{How to cite this article:}

Saida Naik, D. and Chetti, M.B. 2017. Effect of Storage Conditions and Packaging on Sensory Evaluation of Rice. Int.J.Curr.Microbiol.App.Sci. 6(10): 1219-1230. doi: https://doi.org/10.20546/ijcmas.2017.610.147 